\title{
Complicações com uso de esferas não integráveis e integráveis na reconstrução da cavidade anoftálmica
}

\author{
Complications with nonintegrated and integrated spheres in anophthalmic socket \\ reconstruction
}

Silvana Artioli Schellini ${ }^{(1)}$

Erika Hoyama ${ }^{(2)}$

Carlos Roberto Padovani ${ }^{(3)}$

Vera Lúcia R. Ferreira ${ }^{(4)}$

Romualdo Roça ${ }^{(5)}$

\section{RESUMO}

Objetivo: Apresentar as complicações observadas com esferas de diferentes materiais na reconstrução da cavidade anoftálmica.

Métodos: Foram avaliados retrospectivamente 117 portadores de cavidade anoftálmica, com tempo mínimo de seguimento de 6 meses, procurando correlacionar sexo, tipo de esfera utilizada, causa da perda do olho, diâmetro da prótese, tipo de cirurgia e a ocorrência das complicações (deiscências e expulsão da esfera).

Resultados: As deiscências ocorreram principalmente com esferas de polímero vegetal. A expulsão da esfera ocorreu mais precocemente nos implantes integráveis e foi mais freqüente com as esferas nãointegráveis (PMMA). A esfera de polietileno (Polipore) foi a que apresentou menos probabilidade de complicações.

Conclusão: A esfera de polietileno é na atualidade a melhor alternativa para preenchimento da cavidade anoftálmica.

Palavras-chave: Implante de cavidade; Deiscência; Expulsão; Cavidade anoftálmica; Complicações.

\section{INTRODUÇÃOO}

Os relatos da utilização de esferas para reposição do volume perdido, após a remoção do bulbo ou de seu conteúdo, para impedir a contração da cavidade orbitária datam de 1885, com utilização de esferas de vidro por Van Müller ${ }^{1}$.

Após a 2aㅡ Guerra Mundial, as esferas de polimetilmetacrilato (PMMA) substituiram as esferas de vidro, ambas chamadas de esferas (ou inclusões) não integráveis. Na década de 80 houve uma mudança radical dos materiais para confecção das esferas. Surgiram os implantes integráveis como a hidroxiapatita ${ }^{2}$ e o polietileno ${ }^{3}$.

Tanto a hidroxiapatita natural (Bioeye), como o polietileno poroso (POREX), possuem preços impraticáveis em nosso meio. Isso vem motivando estudos que visam ao desenvolvimento de materiais alternativos.

O objetivo deste estudo foi apresentar nossa experiência com a utilização das esferas não integráveis e as integráveis de fabricação brasileira.

\section{MATERIAL E MÉTODO}

Foram avaliados retrospectivamente pacientes portadores de cavidade anoftálmica, operados na Faculdade de Medicina de Botucatu e que recebe- 
ram esferas de polimetilmetacrilato (PMMA), hidroxiapatita associada a polímero vegetal, hidroxiapatita sintética microgranular e polietileno poroso (Polipore).

As cirurgias foram realizadas no período de 1990 até 1998 e o tempo mínimo de seguimento considerado foi de 6 meses. Os pacientes estavam cientes da utilização de esferas dos diferentes tipos de materiais e consentiram com o procedimento.

Observou-se em cada paciente o sexo, o tipo de esfera empregada, a causa da perda do globo, o diâmetro da prótese utilizada, o tipo de cirurgia (enucleação ou evisceração) e a ocorrência das complicações.

Os dados foram submetidos a análise estatística pelos testes de Goodman e Qui-quadrado, procurando-se correlacionar as diversas variáveis estudadas com a ocorrência de complicações como a exposição e expulsão das esferas.

\section{RESULTADOS}

Foram avaliados os dados obtidos de 177 pacientes, a maioria do sexo masculino e com maior percentual dos pacientes recebendo a esfera de PMMA (Tabela 1).

Quanto a causa da perda do bulbo, observou-se predomínio da phthisis bulbi, endoftalmite e trauma (Tabela 2).

As esferas mais utilizadas foram as de diâmetro 17,18 e 19 (Tabela 3).

A maioria dos pacientes foi submetida a evisceração (Tabela 4).

As complicações avaliadas foram a presença de deiscência e expulsão da esfera por entendermos que outras alterações que podem ocorrer após a colocação das esferas não estariam ligadas ao tipo de material utilizado. Assim, observou-se que

\begin{tabular}{|lcccc|}
\hline \multicolumn{4}{|c|}{ Tabela 1. Distribuição de sexo segundo o material da esfera } \\
utilizada - UNESP, 1999. \\
Sexo & \multicolumn{4}{c}{ Freqüência (\%) } \\
\cline { 2 - 5 } & PMMA & P. Vegetal & Hidroxiapatita & Polietileno \\
Feminino & 28,0 & 53,0 & 26,0 & 25,7 \\
Masculino & 72,0 & 47,0 & 74,0 & 74,3 \\
Total & $\mathbf{8 5}$ & $\mathbf{3 4}$ & $\mathbf{2 3}$ & $\mathbf{3 5}$ \\
\hline
\end{tabular}

Tabela 2. Distribuição da causa da perda do globo ocular e o material da esfera utilizada - UNESP, 1999.

\begin{tabular}{lcccc} 
Causa de & \multicolumn{5}{c}{ Freqüência (\%) } \\
\cline { 2 - 5 } Perda & PMMA & P. Vegetal & Hidroxiapatita & Polietileno \\
Phthisis bulbi & 22,4 & 61,8 & 43,5 & 14,3 \\
Endoftalmite & 25,9 & 29,4 & 21,7 & 14,3 \\
Trauma & 14,1 & 13,5 & 13,0 & 28,6 \\
Tumores & 3,5 & 0,0 & 9,0 & 17,1 \\
Glaucoma & 16,5 & 17,6 & 4,0 & 20,0 \\
Outros & 17,6 & 0,0 & 8,8 & 5,7 \\
Total & $\mathbf{8 5}$ & *45 & $\mathbf{2 3}$ & $\mathbf{3 5}$ \\
*Alguns pacientes apresentavam mais que uma causa de perda do globo ocular.
\end{tabular}

\begin{tabular}{|c|c|c|c|c|}
\hline \multicolumn{5}{|c|}{$\begin{array}{c}\text { Tabela 3. Distribuição do diâmetro da esfera utilizada e tipo de } \\
\text { material - UNESP, } 1999 .\end{array}$} \\
\hline \multirow{2}{*}{$\begin{array}{l}\text { Número da } \\
\text { Prótese }\end{array}$} & \multicolumn{4}{|c|}{ Freqüência (\%) } \\
\hline & PMMA & P. Vegetal & Hidroxiapatita & Polietileno \\
\hline 16 & 0,0 & 20,6 & 30,4 & 34,3 \\
\hline 17 & 17,6 & 23,5 & 4,3 & 8,6 \\
\hline 18 & 30,6 & 50,0 & 34,8 & 34,3 \\
\hline 19 & 17,6 & 2,9 & 30,4 & 22,8 \\
\hline 20 & 11,8 & 0,0 & 0,0 & 0,0 \\
\hline Sem informaçãc & o 22,3 & 0,0 & 0,0 & 0,0 \\
\hline Total & 85 & 34 & 23 & 35 \\
\hline
\end{tabular}

\begin{tabular}{|c|c|c|c|c|}
\hline \multirow[b]{2}{*}{ Cirurgia } & \multicolumn{4}{|c|}{ Freqüência (\%) } \\
\hline & PMMA & P. Vegetal & Hidroxiapatita & Polietileno \\
\hline Enucleação & 14,2 & 8,8 & 30,4 & 37,1 \\
\hline Evisceração & 85,8 & 85,3 & 69,6 & 51,4 \\
\hline Ignorado & 0,0 & 5,9 & 0,0 & 11,5 \\
\hline Total & 85 & 34 & 23 & 35 \\
\hline
\end{tabular}

a maioria das complicações ocorreram tardiamente nos pacientes que receberam a esfera de PMMA e precocemente nos que receberam os implantes integráveis (Tabela 5).

A avaliação do percentual de pacientes que apresentaram deiscência de conjuntiva e/ou esclera revelou números semelhantes para as esferas de PMMA (32,9\%), hidroxiapatita microgranular $(35,8 \%)$ e ligeiramente maior com a hidroxiapatita associada ao polímero vegetal (44,1\%); com a esfera de polietileno, o número de deiscências foi menor (11,4\%). Com relação a expulsão da esfera, as de PMMA apresentaram índices significativamente superiores às demais. Das esferas integráveis, a que apresentou maior índice de expulsão foi a de hidroxiapatita associada a polímero vegetal $(20,6 \%)$ (Tabela 6).

\section{DISCUSSÃO}

O material ideal para a confecção de esferas a serem usadas como implantes ou inclusões em cavidades anoftálmicas ainda não é conhecido. Sabe-se que o material deve ser biocompatível, não alergênico e não cancerígeno. Além destas características, é necessário que a esfera tenha preço acessível para que seja empregada em larga escala.

A partir de estudos realizados em animais ${ }^{4-6}$, observou-se que ocorre integração gradual entre o hospedeiro e a esfera, tanto com esferas de polímero vegetal, hidroxiapatita sintética microgranular e o polietileno poroso, sendo iniciado o uso destas esferas em humanos.

Para limitar fatores de erro nas avaliações, determinamos neste estudo o tempo mínimo de acompanhamento como de 6 meses. Porém, com as esferas de PMMA são empregadas há muitos anos, o tempo de observação destes pacientes é maior 


\begin{tabular}{|lcccc|}
\hline \multicolumn{5}{|c|}{$\begin{array}{c}\text { Tabela 5. Distribuição do tempo decorrido para a ocorrência das } \\
\text { complicações segundo o material da esfera utilizada - UNESP, 1999. }\end{array}$} \\
Tempo para & \multicolumn{5}{c}{ Freqüência (\%) } \\
\cline { 2 - 5 } Complicação & PMMA & P. Vegetal & Hidroxiapatita & Polietileno \\
$<2$ meses & 32,1 & 85,0 & 88,9 & 80,0 \\
$>2$ meses & 67,9 & 15,0 & 11,1 & 20,0 \\
Total & $\mathbf{5 6}$ & $\mathbf{2 0}$ & $\mathbf{9}$ & $\mathbf{5}$ \\
\hline
\end{tabular}

do que com as esferas integráveis. Este poderia ser um dos fatores que levou a observação de maior número de complicações nestes pacientes.

A nossa amostra consistiu principalmente de homens, uma vez que estes estão mais sujeitos a traumas, causa importante de perda do bulbo ocular.

Quanto ao tamanho, utilizou-se esferas com diâmetro variando de 16 a $19 \mathrm{~cm}$, justamente os mais adequados para este fim.

Os fatores apontados: cirurgia realizada, tipo de cavidade, época de colocação do implante, tamanho e envoltório da esfera podem estar associados a complicações no pós-operatório ${ }^{7}$, tendo sido por este motivo avaliados.

As complicações observadas foram deiscência conjuntival que, quando pequena pode evoluir para fechamento espontâneo, sendo conseqüência, principalmente quando ocorre precocemente, de falha técnica na sutura, como foi o caso em vários de nossos pacientes.

A deiscência conjuntival pode ser acompanhada de esclera com exposição do implante e pode ser observada com todos os tipos de esferas que utilizamos, com maior proporção para as esferas de polímero vegetal. Nossas observações permitem apontar o fator causador das deiscências no caso da esfera se polímero vegetal, como sendo a quantidade importante de espículas que estas esferas possui. Já, no caso das esferas de PMMA, as contraturas da cavidade, assim como os traumas da superfície provocados por próteses externas mal adaptadas, poderiam ser os fatores, acrescidos ainda pelo longo tempo de observação.

A deiscência da conjuntiva pode ocorrer também com o uso de hidroxiapatita natural e com esferas FCI, tipo de hidroxiapatita sintética desenvolvida na França9.

Embora esferas de silicone tenham sido relacionadas a menor índice de exposição do que as de hidroxiapatita natural ${ }^{10}$, "Survey" feito entre filiados da Sociedade de Oftalmoplástica americana, apontou possibilidade de extrusão em $1,7 \%$ dos pacientes que receberam hidroxiapatita natural contra 5,6\% dos que receberam esferas de silicone ${ }^{11}$.
A exposição do implante de hidroxiapatita natural ocorre por: infecção, tensão na sutura, tamanho exagerado da esfera, localização superficial e inadequada do implante, falha técnica, espículas e fatores químicos ${ }^{12}$.

A exposição das esferas, principalmente de hidroxiapatita microgranular, esteve associada a formação de granulomas.

As exposições, em geral, provocam liquefação e necrose dos implantes de hidroxiapatita natural ${ }^{10} \mathrm{e}$ também nas de polímero vegetal e hidroxiapatita microgranular que utilizamos, desenvolvendo, os pacientes, excessiva quantidade de secreção de odor fétido. Este tipo de reação não foi observado com o polietileno poroso.

A expulsão espontânea da esfera pode ocorrer com as esferas de PMMA, como observamos em vários pacientes. Porém, com as esferas integráveis este fato só ocorre em fases muito precoces do período pós-operatório e nunca depois que já houve integração esfera-hospedeiro. As expulsões precoces, em geral, decorrem de falhas técnicas ocorridas no ato operatório e independem das esferas utilizadas.

Estamos assistindo a mudanças no tratamento da cavidade anoftálmica. Apesar dos vários estudos realizados, as esferas deverão ainda passar por período de observação mais longo para que se decida pelo melhor material a ser empregado. De qualquer forma, com os dados obtidos neste estudo, dentre as 4 esferas utilizadas, a melhor opção seria a de polietileno poroso pelo menor índice de complicações observadas.

\section{SUMMARY}

Purpose: To observe the complications with spheres of different types of material used in anophthalmic cavity reconstruction.

Methods: 117 patients with anophthalmic cavity were retrospectivelly studied. The minimum follow-up was 6 months. We correlated sex, type of used sphere, causes of eye loss, sphere diameter, surgery done and complications (dehiscence and sphere expulsion).

Results: Dehiscence occurred mainly with vegetal polymer spheres. The integrated implants had earlier sphere expulsion than those nonintegrated and the latter extruded more than the others. The polyethylene sphere (Polipore) presented less complications than the others.

Conclusion: Nowadays we consider the polyethylene sphere the best choice to fill the anophthalmic socket.

\begin{tabular}{|c|c|c|c|c|c|c|c|c|}
\hline \multirow{3}{*}{ Complicação } & \multicolumn{8}{|c|}{ Freqüência (\%) } \\
\hline & \multicolumn{2}{|c|}{ PMMA } & \multicolumn{2}{|c|}{ P. Vegetal } & \multicolumn{2}{|c|}{ Hidroxiapatita } & \multicolumn{2}{|c|}{ Polietileno } \\
\hline & ABS. & REL. & ABS. & $\overline{\text { REL. }}$ & ABS. & $\overline{\text { REL. }}$ & ABS. & REL. \\
\hline Deiscência & 28 & 32,9 & 15 & 44,1 & 8 & 34,8 & 4 & 11,4 \\
\hline Expulsão & 28 & 32,9 & 7 & 20,6 & 1 & 4,3 & 1 & 2,9 \\
\hline Total & $56 / 85$ & 65,9 & $22 / 34$ & 64,7 & $9 / 23$ & 39,1 & $5 / 35$ & 14,3 \\
\hline
\end{tabular}


Keywords: Cavity implant; Dehiscence; Expulsion; Anophthalmic socket; Complications.

\section{REFERÊNCIAS BIBLIOGRÁFICAS}

1. Tonkelaar J, Henkes HE, Leersun GK. A short history of the artificial eye. Doc Ophthalmol 1991;77:349-54.

2. Perry AC. Integrated orbital implants. Adv Ophthal Plast Reconstr Surg 1988;8:75-80.

3. Rubin PAD, Popham JK, Bilyk JR, Shore JW. Comparison of fibrovascular ingrowth into hydroxyapatite and porous polyethylene orbital implants. Ophthal Plast Reconstr Surg 1994;10:96-103.

4. Schellini SA, Marques MEA, Rahal SC et al. Vegetal polymer in anophthalmic socket reconstruction. Invest Ophthal Vis Sci 1996;37:S616.

5. Schellini SA, Marques MEA, Rahal SC et al. Synthetic hydroxyapatite in anophthalmic socket reconstruction. Invest Ophthal Vis Sci 1998;39:S502.
6. Schellini SA, Marques MEA, Taga EM, Roça R, Padovani CR. Comparison between synthetic hydroxyapatite and porous polythylene in eviscerated cavities of rabbits. Invest Ophthal Vis Sci 1999;40:S890.

7. Rodrigues AC, Schellini SA, Moraes-Silva MRB, Padovani CR. Fatores relacionados à extrusão do implante de cavidade. Rev bras oftalmol 1996;56:259-64.

8. Buettner H, Bartley GB. Tissue breakdown and exposure associated with orbital hydroxyapatite implants. Am J Ophthalmol 1992;113:669-73.

9. Jordan DR, Munro SM, Mrowntein S et al. A synthetic hydroxyapatite implant: the so called Counterfield implant. Ophthal Plast Reconstr Surg 1998;14:244-9.

10. Nunery WR, Heing GW, Bonnin JM et al. Exposure rate of hydroxyapatite spheres in the anophthalmic socket. Ophthal Plast Reconstr Surg 1993; 9:96-104.

11. Hornblass A, Biesman BS, Eviatar JA. Current techniques of enucleation: a survey of 5439 intraorbital implants and a review of the literature. Ophthal Plast Reconstr Surg 1995;11:77-88.

12. Goldberg RA, Holds JB, Ebrahimpour J. Exposed hydroxyapatite orbital implants. Ophthalmology 1992;99:831-6.

\title{
IV CURSO DE TREINAMENTO TÉCNICO E CIENTÍFICO EM BANCO DE OLHOS DA APABO
}

\author{
31 de Julho a 11 de Agosto de 2.000 \\ ESCOLA PAULISTA DE MEDICINA - SP
}

Promoção: Associação Pan-Americana de Banco de Olhos e Departamento de Oftalmologia da UNIFESP-EPM

Informações: Dr. Elcio H. Sato (Presidente da APABO Brasil)

Tel: (11) 284-4033 • e-mail: cornea@pobox.com

Srta. Ana Maria Guimarães (Secretária da APABO Brasil)

e-mail: apabo@united.com.br 